\title{
Unraveling the packaging mechanism of coronavirus ribonucleocapsid
}

Volume 5 Issue 2 - 2017

\section{Tai-huang Huang}

Institute of Biomedical Science, Academia Sinica, Republic of China

Correspondence: Tai-huang Huang, Institute of Biomedical Science, Academia Sinica, Taiwan, Republic of China; Email taihuanghuang@gmail.com

Received: March 01, 2017 | Published: March 15, 2017 in $2012 .{ }^{1}$ making $\mathrm{CoV}$ as an important human pathogen in the $21 \mathrm{st}$ century. ${ }^{1}$ The emergence of SARS evoked a world-wide interest in research on coronavirus and resulted in impressive progress in our understanding of its structure and function (For a review see Hilgenfeld and Perris 2013). ${ }^{1}$ The CoV nucleocapsid protein N is the most abundant structural protein in the $\mathrm{CoV}$ infected cells. Its primary function is to package the $\sim 30 \mathrm{~kb}$ single stranded, 5'-capped positive strand viral genome RNA molecule into a ribonucleoprotein (RNP) complex called the capsid. RNP packaging is a fundamental part of viral self-assembly and the RNP complex constitutes the essential template for replication by the RNA-dependent RNA polymerase complex.

EM studies of coronaviruses have shown that coronavirus RNPs are helical, consisting of coils of 9-16 $\mathrm{nm}$ in diameter and a hollow interior of approximately 3-4 $\mathrm{nm}$ (For a review see Chang and Huang, 2014). ${ }^{2}$ Accommodation of the $\sim 30 \mathrm{~kb} \mathrm{CoV}$ genome into the newly formed virion spherules $<100 \mathrm{~nm}$ in size necessitates an extremely well-packed, largely helical, supercoiling of the nucleic acid within the RNP core. The pleomorphic nature of the coronavirus particle has hampered the effort to obtain high-resolution virion image at atomic resolution. The inability to observe a well-structured RNP layer inside the SARS-CoV particle and only short coiled fragments of RNP in MHV in the cryo-EM reconstructions strongly suggests that the helical nucleocapsid is a very flexible structure that extensively twists and folds upon itself. ${ }^{3}$ Extensive biophysical studies suggest that all $\mathrm{CoV} \mathrm{N}$ proteins share the same modular organization containing two structured domains, the N-terminal domain (NTD) and the C-terminal domain (CTD), surrounded by three intrinsically disordered regions (IDR) called the $\mathrm{N}$-terminal arm $(\mathrm{N}$-arm), the central linker region (LKR), and the $\mathrm{C}$-terminal tail (C-tail), respectively. ${ }^{4} \mathrm{~N}$ protein forms a dimer, which constitutes the basic building block of the nucleocapsid, through its CTD.$^{4,5}$ Studies from several laboratories have established that the NTD is involved in RNA binding, whereas the CTD is involved in RNA binding and oligomerization. All three IDRs of coronaviral N protein can modulate the RNA-binding and oligomerization properties of NTD and CTD, respectively. ${ }^{6}$ In addition, the LKR of the N protein with Ser-Arg-rich sequences has also been shown to contain an RNAbinding region and putative phosphorylation sites that might regulate $\mathrm{N}$ protein functions. ${ }^{7,8}$ and $\mathrm{N}-\mathrm{M}$ interaction. No existing data supports the presence of a long-lived SARS-CoV N oligomer or intermediate in solution and the SARS-CoV genomic ssRNA by itself is unlikely to exist as a helix of the length observed in cryo-EM. Thus, packaging of SARS-CoV RNP proceeds most likely through a RNA bindingcoupled packaging mechanism. This suggests that coronavirus RNA synthesis is coupled to the encapsidation of nascent RNA, The modular organization with three long IDRs provides the $\mathrm{N}$ protein

with considerable flexibility, as also the RNA molecule. Based on available detailed 3D structural information of the $\mathrm{N}$ protein modules and our understanding of N-RNA interaction we proposed a probable CoV RNP packaging model derived from the crystal structure of the CTD. ${ }^{9}$ which was shown to exist transiently in solution by disulfide trap experiment. ${ }^{10}$

A putative scenario of the molecular events leading to the formation of RNP may involve the following steps: Initial binding:

I. RNA first binds at either NTD or CTD which facilitates binding of other modules to RNA in a coupled-allostery manner. RNA molecule threads in between the two structural domains. This initial N-RNA binary complex (RNP0) may have several $\mathrm{N}$ protein bound at a particular time.

II. Growth: The RNP0 could grow by either recruiting more N to the adjacent RNA sites, or it could slide or hop along the linear RNA molecule and combine with other smaller N-RNA oligomers to form a larger oligomer (RNPN) of various sizes. RNPN would pack in a structure with CTD forming the helical core and RNA wraps and twists around the helical groove through mostly electrostatic interaction between the positively charge residues in the groove and the phosphate backbone of the RNA molecule.

III. Packaging of NTD: The NTD module will cap on the outside of the helical CTD-RNA complex with the positively charged surface covering the free phosphate groups of the RNA molecule. Furthermore, RNA bases sticking out of the CTD groove could intercalate in between the aromatic rings at the bottom of the $\beta$-sheet on the NTD core. The presence of IDRs permits the two structural domains considerable freedom to adapt a wide range of orientations and positions for optimal packing of the RNP complex. Likewise, the RNA molecule can adjust to local conformation by an induced-fit process. 
IV. Thermodynamic basis: Electrostatic interaction drives the formation of N-RNA complex but the multitude of weak proteinprotein interactions contributes towards the self-assembly of the helical RNP.

The RNP structure proposed above would have an outer diameter of $\sim 16 \mathrm{~nm}$ and an inner diameter of $\sim 4 \mathrm{~nm}$, consistent with that observed by cryo-EM. Each N dimer would bind to 7 RNA bases. The combination of a modular structure incorporating IDRs, multiple sites of moderate RNA binding affinity, and weak dimer-dimer interaction in the N protein not only allows the packaging of a stable RNP but also offers an energetically favorable condition for the expression of the viral genomic information through an unzipping mechanism for unwinding and dissociation of the viral RNA molecule from the $\mathrm{N}$ protein in a stepwise manner, one module at a time. This will avoid the need to overcome a high-energy barrier of dissociating a whole $\mathrm{N}$ protein at once. Recent studies suggest that $\mathrm{N}$ proteins of corona viruses and other viruses could be useful antiviral drug targets against infections caused by these viruses because they serve many crucial functions during the viral lifecycle. ${ }^{11}$ The advent in understanding the Co V RNP packaging could aid the development of $\mathrm{N}$ protein targeted drug. Nonetheless, more work to verify and refine the proposed RNP packaging model is needed.

\section{Acknowledgments}

None.

\section{Conflicts of interest}

None.

\section{References}

1. Hilgenfeld $\mathrm{R}$, Peiris $\mathrm{M}$ From SARS to MERS: 10 years of research on highly pathogenic human coronaviruses. Antiviral Res. 2013;100(1):286-295.

2. Chang $\mathrm{C}-\mathrm{k}$, Hou $\mathrm{M}-\mathrm{H}$, Chang $\mathrm{C}-\mathrm{F}$ The SARS coronavirus nucleocapsid protein -Forms and functions. Antiviral Research. 2014;103:39-50.
3. Bárcena M, Oostergetel GT, Bartelink W et al. Cryo-electron tomography of mouse hepatitis virus: Insights into the structure of the coronavirion. Proceedings of the National Academy of Sciences. 2009;106(2):582-587.

4. Chang CK, Sue SC, Yu TH et al. Modular organization of SARS coronavirus nucleocapsid protein. Journal of Biomedical Science. 2006;13(1):59-72.

5. Yu IM, Oldham ML, Zhang J, Chen J Crystal Structure of the Severe Acute Respiratory Syndrome (SARS) Coronavirus Nucleocapsid Protein Dimerization Domain Reveals Evolutionary Linkage between Corona- and Arteriviridae. J Biol Chem. 2006;281(25):17134-17139.

6. Chang CK, Hsu YL, Chang YH et al. Multiple nucleic acid binding sites and intrinsic disorder of severe acute respiratory syndrome coronavirus nucleocapsid protein: implications for ribonucleocapsid protein packaging. J Virol. 2009;83(5):2255-2264.

7. Peng T-Y, Lee $\mathrm{K}-\mathrm{R}$, Tarn $\mathrm{W}-\mathrm{Y}$ Phosphorylation of the arginine/ serine dipeptide-rich motif of the severe acute respiratory syndrome coronavirus nucleocapsid protein modulates its multimerization, translation inhibitory activity and cellular localization. FEBS. 2008;275(16):4152-4163.

8. Surjit M, Kumar R, Mishra RN et al. The Severe Acute Respiratory Syndrome Coronavirus Nucleocapsid Protein Is Phosphorylated and Localizes in the Cytoplasm by 14-3-3-Mediated Translocation. Journal of Virology. 2005;79(17):11476-11486.

9. Chen CY, Chang CK, Chang YW et al. Structural of the SARS coronavirus nucleocapsid protein RNA-binding dimerization domain suggests a mechnism for helical packaging of viral RNA. J Mol Biol. 2007;368(4):1075-1086.

10. Chang CK, Chen CM, Chiang MH et al. Transient oligomerization of the SARS-CoV N protein-implication for virus ribonucleoprotein packaging. Plos One. 2013;8(5):e65045.

11. Chang CK, Lo SC, Wang YS, Hou MH Recent insights into the development of therapeutics against coronavirus diseases by targeting $\mathrm{N}$ protein. Drug discovery today. 2016;21(4):562-572. 